Mathematical Sciences and Applications

E-NOTES

MATHEMATICAL

SCIENCES

AND APPLICATIONS

E-NOTES

https://doi.org/10.36753/mathenot.626461

9 (2) 64-73 (2021) - Research Article

ISSN: 2147-6268

(C)MSAEN

\title{
Investigation of Exact Solutions of some Nonlinear Evolution Equations via an Analytical Approach
}

\author{
Meryem Odabaşı Köprülü
}

\begin{abstract}
This study investigates exact analytical solutions of some nonlinear partial differential equations arising in mathematical physics. To this reason, the Kudryashov-Sinelshchikov equation, the ZK-BBM equation and the Gardner equation have been considered. With the implementation of the trial solution algorithm, solitary wave, bright, dark and periodic exact traveling wave solutions of the considered equations have been attained. The solutions have been checked and graphs have been given via package programs to see the behavior of the waves.
\end{abstract}

Keywords: Nonlinear evolution equations; Travelling wave solutions; Analytical methods.

AMS Subject Classification (2020): Primary: 35C07; Secondary: 35Q99.

\section{Introduction}

Investigating exact solutions of nonlinear partial differential equations still take a great deal of attention due to the success of explaining the physical phenomenon. Nonlinear equations (NLEs) arise in many areas such as biology, chemistry, solid state physics, nonlinear optics, fluid mechanics, quantum field theory, and so on. In this work, considered models are Kudryashov-Sinelshchikov equation, Zakharov-Kunzetsov-Benjamin-Bona-Mahony equation and Gardner equation.

Nonlinear differential equations like Korteweg-de Vries $(\mathrm{KdV})$ and Burgers describe the solitary and periodic waves in a mixture of gas and liquid bubbles. Kudryashov and Sinelshchikov [11], [12]proposed a more general model for describing pressure waves in a a gas and liquid bubbles mixture by taking into account of the heat transfer and the viscosity of liquid. The equation is known as Kudryashov-Sinelshchikov (KS) equation [16].

Benjamin et al. [3] proposed Zakharov-Kunzetsov-Benjamin-Bona-Mahony (ZK-BBM) equation to be an alternative model for the KdV equation for long waves. It has applications in nonlinear dispersive systems, drift waves in a plasma, and waves in rotating fluids [7].

Gardner equation has been proposed by Miura about half a century ago [18]. It is an expansion of the KdV equation and has identical features with $\mathrm{KdV}$ equation, but also expands the order of availability. Unlike the KdV equation which represents shallow water waves, Gardner equation construct a model for deep ocean waves [4], [9], 
[24]. The equation has crucial applications in different fields such as quantum field theory, fluid dynamics, solid state physics, and plasma physics [5], [10].

Because of the applications mentioned, scientists still study NLEs and their solution methods. Numerous analytical and numerical methods have been proposed and applied to NLEs up to now. Recently, based on the approach proposed by Ma and Fuchssteiner [17], Cheng-Shi Liu [13]- [15] introduced the trial solution method that reduces the undertaking equation to solvable differential equations for solving nonlinear differential equations. Some authors [6], [19-23] applied this efficient procedure to some nonlinear evolution equations. In this study, among the methods used for nonlinear partial differential equations, the trial solution procedure is considered. The method have been applied to the KS, ZK-BBM and Gardner equations for the first time and exact analytical solutions of them have been attained.

\section{Trial Solution Procedure}

To summarize the solution procedure of the method we can use four steps [13]- [15]:

Step 1. A nonlinear evolution equation is given as

$$
V\left(u, u_{x}, u_{t}, u_{x x}, u_{t t}, u_{x t}, \ldots\right)=0 .
$$

Introducing the wave transformation

$$
u(x, t)=U(\xi), \quad \xi=x-\rho t,
$$

reduces Eq. (2.1) to an ODE as follows:

$$
N\left(U, U^{\prime}, U^{\prime \prime}, \ldots\right)=0
$$

Step 2. Auxiliary equation which is an important step of the method is given by

$$
\left(U^{\prime}\right)^{2}=H(U)=\sum_{i=0}^{n} a_{i} U^{i},
$$

which has the essential coefficients $a_{i}, i=1, \ldots, n$. Substituting the trial equation and other derivatives into Eq. (2.2) yields a polynomial:

$$
\Omega(U)=p_{s} U^{n}+\ldots+p_{1} U+p_{0} .
$$

With the aid of balancing procedure, one can find $n$.

Step 3. If one lets all coefficients of the polynomial to zero, it gives

$$
p_{i}=0, \quad i=0, \ldots, s .
$$

The requisite constants $a_{0}, \ldots, a_{n}$ can be found by solving the system above.

Step 4. By integrating Eq. (2.3), the important equation of the solution procedure can be imparted with

$$
\pm\left(\xi-\xi_{0}\right)=\int \frac{1}{\sqrt{H(U)}} d U
$$

Analytical solutions of Eq. (2.1) are recovered when discriminant of $H(U)$ is classified.

\section{Application of the Method}

Example 3.1. Consider the Kudryashov-Sinelshchikov equation [8], [16]:

$$
u_{t}+\gamma u u_{x}+u_{x x x}-\left(u u_{x x}\right)_{x}-\beta u_{x} u_{x x}=0,
$$


where $\gamma, \beta$ are real parameters and $u$ is the density.

Using

$$
u(x, t)=U(\xi), \quad \xi=x-\rho t,
$$

Eq. (3.1) turns into the form of an ODE:

$$
-(\rho U)^{\prime}+\gamma\left(\frac{U^{2}}{2}\right)^{\prime}-\left(U U^{\prime \prime}\right)^{\prime}+U^{\prime \prime \prime}-\frac{\beta}{2}\left[\left(U^{\prime}\right)^{2}\right]^{\prime}=0 .
$$

Integrating this equation once

$$
-\rho U+\gamma \frac{U^{2}}{2}-U U^{\prime \prime}+U^{\prime \prime}-\frac{\left(U^{\prime}\right)^{2} \beta}{2}+\mu=0,
$$

where $\mu$ is constant of integration.

Applying the balance procedure in Eq. (3.3) gives $n=2$.

Then, the trial equation can be chosen as (also known as Riccati equation)

$$
\left(U^{\prime}\right)^{2}=a_{0}+a_{1} U+a_{2} U^{2} .
$$

We will find the solution of this equation by using the trial solution algorithm and complete discrimination system for polynomials. The overdetermined equations derived from the coefficients of the polynomial are as follows:

$$
\begin{gathered}
a_{0} \beta+a_{1}+2 \mu=0 \\
-\left(1+\beta a_{1}\right)+2 a_{2} a_{2}-2 \rho=0 \\
-(2+\beta) a_{2}+\gamma=0 .
\end{gathered}
$$

Following coefficients are obtained:

$$
\begin{gathered}
a_{0}=\frac{2\left(-2 \rho-\beta \rho+2 \mu+3 \beta \mu+\beta^{2} \mu+\gamma\right)}{(2+\beta)(1+\beta) \beta} \\
a_{1}=-\frac{2(2 \rho+\beta \rho-\gamma)}{(2+\beta)(1+\beta)} \\
a_{2}=\frac{\gamma}{2+\beta} .
\end{gathered}
$$

Inserting the Eqs. (3.4) in the integral form Eq. (2.4), we obtain

$$
\pm\left(\xi-\xi_{0}\right)=\int \frac{1}{\sqrt{\frac{2\left(-2 \rho-\beta \rho+2 \mu+3 \beta \mu+\beta^{2} \mu+\gamma\right)}{(2+\beta)(1+\beta) \beta}-\frac{2(2 \rho+\beta \rho-\gamma)}{(2+\beta)(1+\beta)} U+\frac{\gamma}{2+\beta} U^{2}}} d U .
$$

Integrating Eq. (3.5), we obtain

$$
U(\xi)=\frac{-2 a_{1}+e^{-\sqrt{a 2}\left(\xi+\xi_{0}\right)}\left(\left(a_{1}^{2}-4 a_{0} a_{2}\right) e^{2 \sqrt{a 2} \xi}+e^{2 \sqrt{a_{2}} \xi_{0}}\right)}{4 a_{2}} .
$$

Using the transformations (3.2), the solution (3.6) reduces to the traveling wave solution to the KudryashovSinelshchikov equation as:

$$
u(x, t)=\frac{b \rho+2 \rho-\gamma}{(1+b) \gamma}+\cosh \sqrt{\frac{\gamma}{2+\beta}}(x-\rho t),
$$

and

$$
u(x, t)=\frac{b \rho+2 \rho-\gamma}{(1+b) \gamma}+\sinh \sqrt{\frac{\gamma}{2+\beta}}(x-\rho t),
$$

when $\gamma(2+\beta)>0$. 

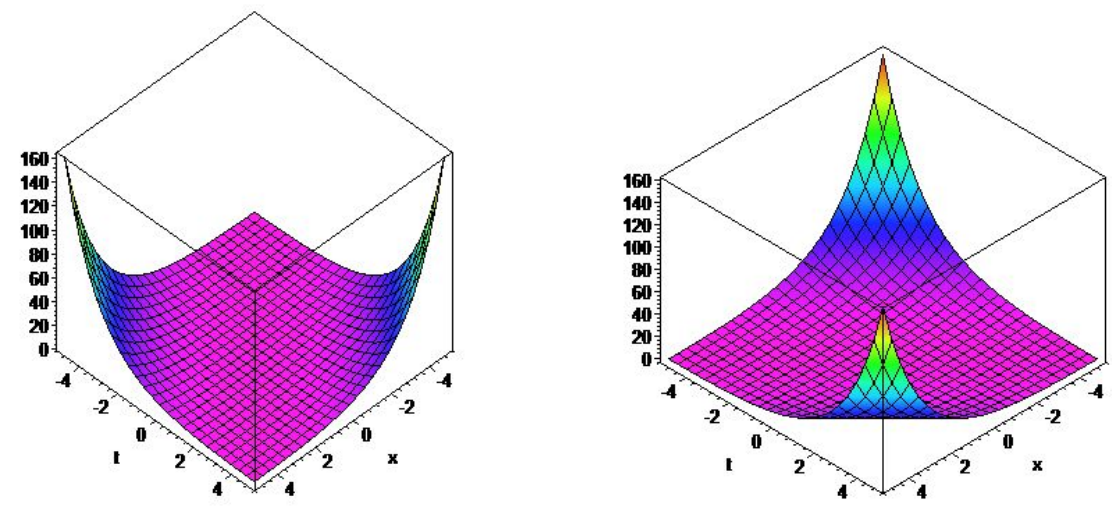

Figure 1. Solution (3.7) for $\gamma=\beta=a=b=1$ and $\rho= \pm 1$, respectively.
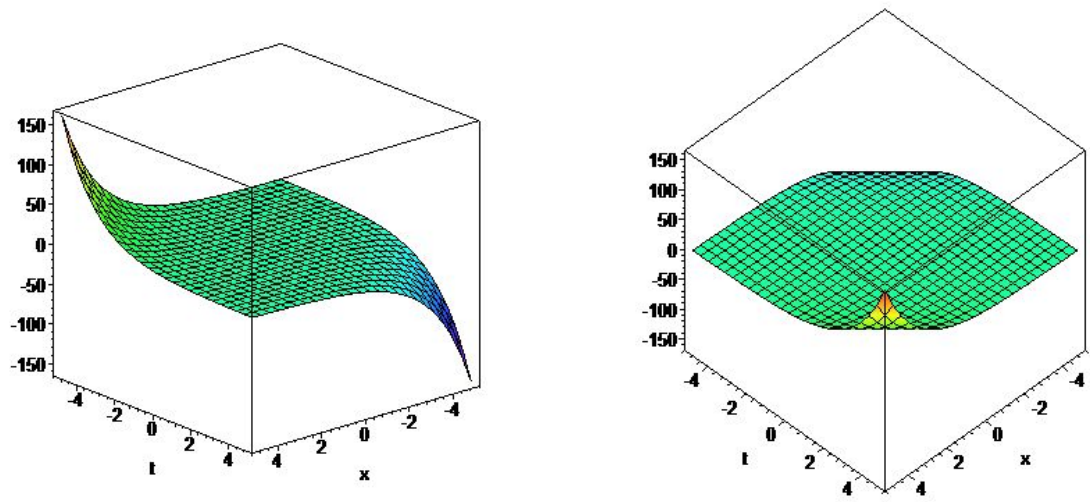

Figure 2. Solution (3.8) for $\gamma=\beta=a=b=1$ and $\rho= \pm 1$, respectively.

The solutions mean periodic solutions when $\gamma(2+\beta)<0$ :

$$
u(x, t)=\frac{b \rho+2 \rho-\gamma}{(1+b) \gamma}+\cos \sqrt{\frac{-\gamma}{2+\beta}}(x-\rho t),
$$

and

$$
u(x, t)=\frac{b \rho+2 \rho-\gamma}{(1+b) \gamma}+\sin \sqrt{\frac{-\gamma}{2+\beta}}(x-\rho t) .
$$

Figures 1- 4 show the behavior of the solutions (3.7)- (3.10) for some arbitrary constants.

Example 3.2. Consider the ZK-BBM equation [1], [3], [7]:

$$
u_{t}+u_{x}-2 \alpha u u_{x}-\beta u_{x x t}=0,
$$

where $\alpha$ and $\beta$ are arbitrary constants.

Some authors have studied different forms of the ZK equation such as with power law nonlinearity [2] and space-time fractional modified KdV-ZK equation [25] by means of the Bernoulli sub-equation function method. 

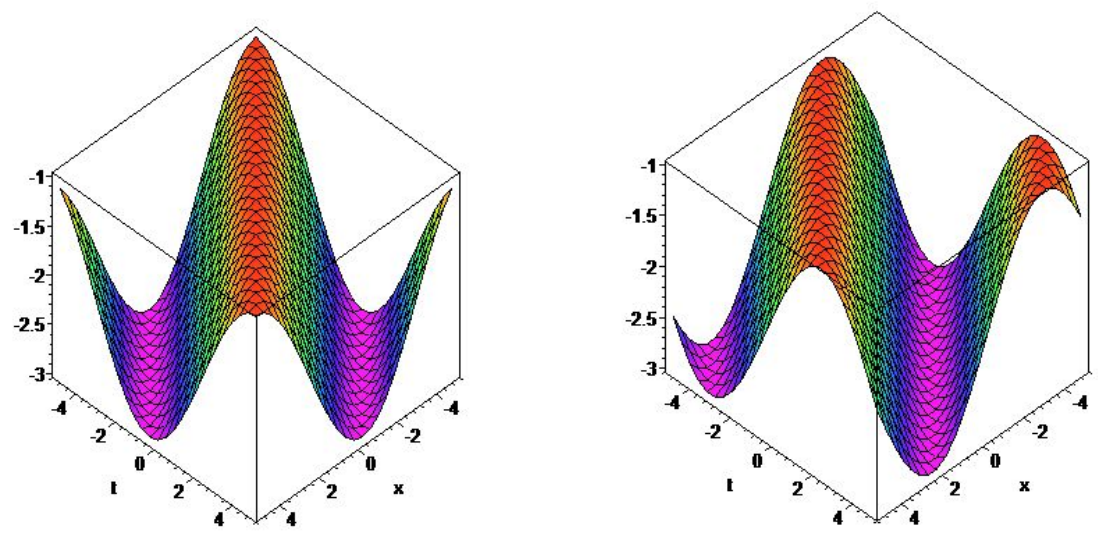

Figure 3. Solutions (3.9) and (3.10) for $\gamma=-1, \beta=a=b=\rho=1$, respectively.
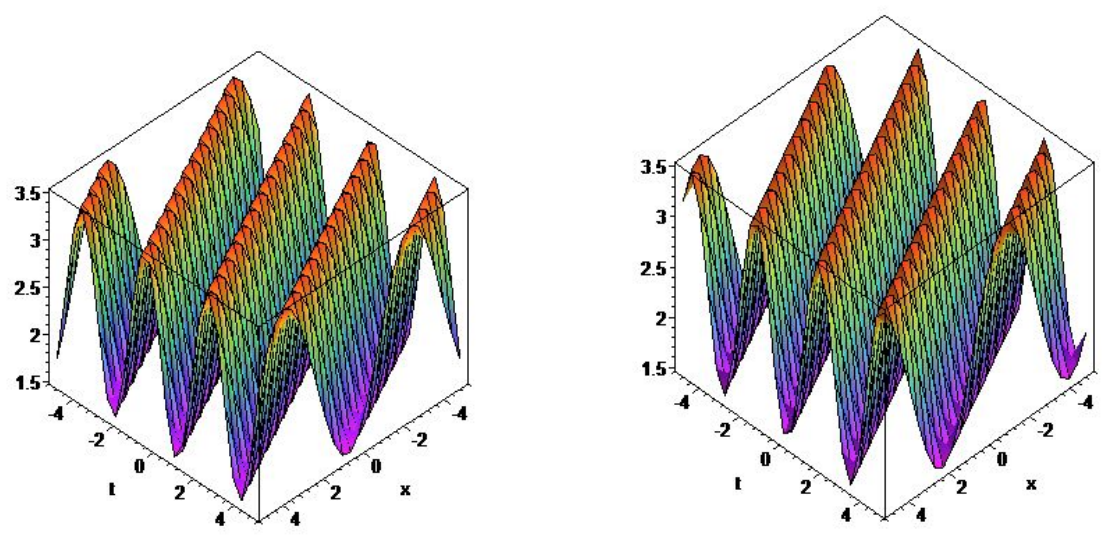

Figure 4. Solutions (3.9) and (3.10) for $\gamma=1, \beta=-3, a=b=1, \rho=2$, respectively.

Using the transformations (3.2) change Eq. (3.11) into the form of an ODE:

$$
\beta \rho U^{\prime \prime \prime}-2 \alpha U U^{\prime}+(1-\rho) U^{\prime}=0 .
$$

Integrating Eq. (3.12) with respect to $\xi$ yields

$$
\beta \rho U^{\prime \prime}-2 \alpha U^{2}+(1-\rho) U+\mu=0,
$$

where $\mu$ is constant of integration.

Balancing the terms $U^{2}$ and $U^{\prime \prime}$, one can have $n=3$.

$$
\left(U^{\prime}\right)^{2}=a_{0}+a_{1} U+a_{2} U^{2}+a_{3} U^{3} .
$$

Substituting Eq. (3.14) and other derivatives into Eq. (3.13), and letting the coefficients of obtained polynomial zero, we have

$$
\begin{gathered}
2 \mu+\beta \rho a_{1}=0 \\
1-\rho+\beta \rho a_{2}=0 \\
-2 \alpha+3 \beta \rho a_{3}=0 .
\end{gathered}
$$

Following coefficients are determined from the solution of the system: 


$$
a_{0}=a_{0}, \quad a_{1}=-\frac{2 \mu}{\beta \rho}, \quad a_{2}=\frac{-1+\rho}{\beta \rho}, \quad a_{3}=\frac{2 \alpha}{3 \beta \rho} .
$$

Substituting these coefficients into Eq. (2.4) by assuming $a_{1}=0$, we have

$$
\pm\left(\xi-\xi_{0}\right)=\int \frac{1}{\sqrt{a_{0}+\frac{\rho-1}{\beta \rho} U^{2}+\frac{2 \alpha}{3 \beta \rho} U^{3}}} d U .
$$

Integrating Eq. (3.15) by choosing $a_{0}=0$, we obtain bright soliton solution:

$$
u(x, t)=\frac{3(1-\rho)}{2 \alpha} \operatorname{sech}^{2}\left[\frac{\sqrt{\rho-1}}{2 \sqrt{\beta c}}(x-\rho t)\right],
$$

and singular soliton solution

$$
u(x, t)=\frac{3(\rho-1)}{2 \alpha} \operatorname{csch}^{2}\left[\frac{\sqrt{\rho-1}}{2 \sqrt{\beta c}}(x-\rho t)\right] .
$$

The solutions can be expressed in the forms:

$$
u(x, t)=\frac{3(1-\rho)}{\alpha\left(\cos \left[\sqrt{\frac{1-\rho}{\beta \rho}}(x-\rho t)\right]+1\right)},
$$

and

$$
u(x, t)=\frac{3(1-\rho)}{\alpha\left(\sin \left[\sqrt{\frac{1-\rho}{\beta \rho}}(x-\rho t)\right]+1\right)} .
$$
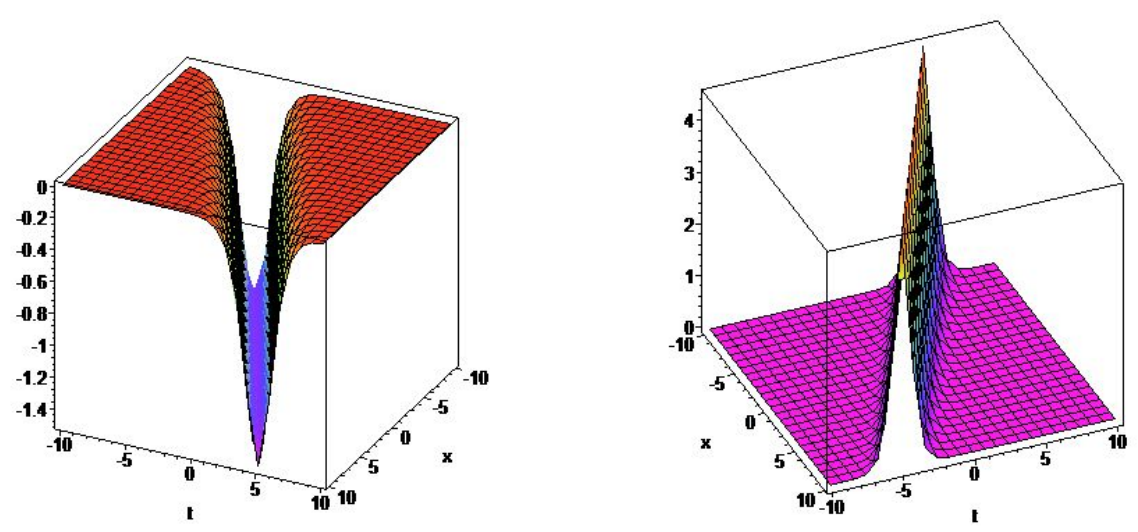

Figure 5. Solution (3.16) for $\alpha=\beta=1, \rho= \pm 2$, respectively.

Figure 5 shows the soliton solution (3.16) for some chosen constants.

Example 3.3. Consider the Gardner equation [5], [18]:

$$
u_{t}+\alpha u u_{x}+\beta u^{2} u_{x}+\gamma u_{x x x}=0
$$

where $\alpha, \beta, \gamma$ are arbitrary constants.

The transformations (3.2) turns the Eq. (3.17) to an ODE in the form:

$$
\gamma U^{\prime \prime \prime}-\rho U^{\prime}+\alpha U U^{\prime}+\beta U^{2} U^{\prime}=0 .
$$


If one integrates the Eq. (3.18) with respect to $\xi$, it gives

$$
\gamma U^{\prime \prime}-\rho U+\frac{\alpha}{2} U^{2}+\frac{\beta}{3} U^{3}+\mu=0,
$$

where $\mu$ is constant of integration.

Balancing terms $U^{\prime \prime}$ and $U^{3}$, one can find $n=4$.

$$
\left(U^{\prime}\right)^{2}=a_{0}+a_{1} U+a_{2} U^{2}+a_{3} U^{3}+a_{4} U^{4} .
$$

Substituting derivatives obtained from Eq. (3.20) into Eq. (3.19), and letting the coefficients of obtained polynomial zero, we have

$$
\begin{aligned}
& 2 \mu+\gamma a_{1}=0 \\
& -\rho+\gamma a_{2}=0 \\
& \alpha+3 \gamma a_{3}=0 \\
& \beta+6 \gamma a_{4}=0 .
\end{aligned}
$$

Coefficients are as follows:

$$
a_{0}=a_{0}, \quad a_{1}=-\frac{2 \mu}{\gamma}, \quad a_{2}=\frac{\rho}{\gamma}, \quad a_{3}=-\frac{\alpha}{3 \gamma}, \quad a_{4}=-\frac{\beta}{6 \gamma} .
$$

By inserting Eqs. (3.21) into Eq. (2.4), one obtain

$$
\pm\left(\xi-\xi_{0}\right)=\int \frac{1}{\sqrt{a_{0}-\frac{2 \mu}{\gamma} U+\frac{\rho}{\gamma} U^{2}+\frac{\alpha}{3 \gamma} U^{3}-\frac{\beta}{6 \gamma} U^{4}}} d U .
$$
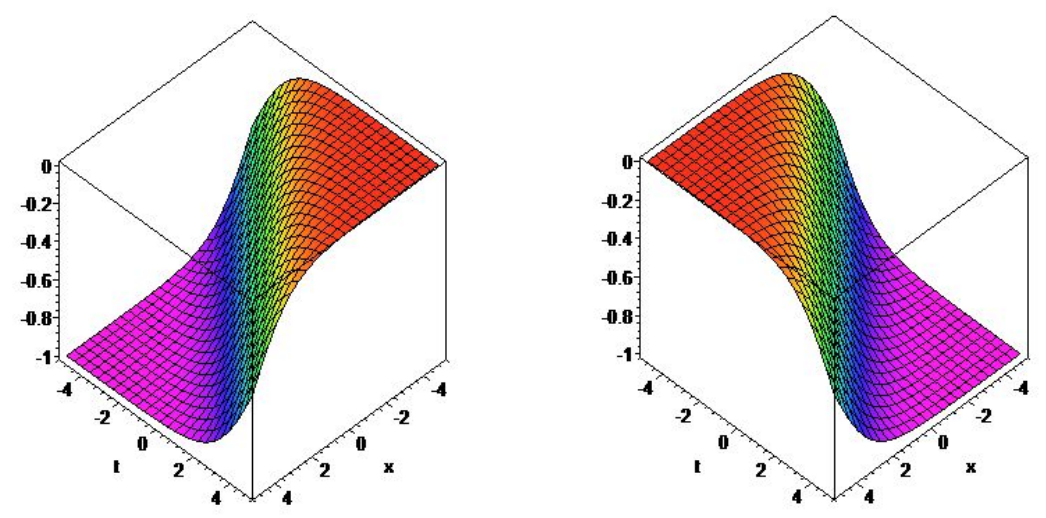

Figure 6. Solution (3.23) for $\alpha=\beta=\rho=1$, respectively.

Integrating Eq. (3.22) by choosing $a_{0}=0, a_{2}=-\frac{\alpha^{2}}{6 \beta \gamma}$ and $\mu=0$, we obtain 1-soliton solution:

$$
u(x, t)=\frac{-\alpha}{2 \beta}\left(1 \pm \tanh \left[\frac{\sqrt{\rho}}{2 \sqrt{\alpha}}(x-\rho t)\right]\right),
$$

and solitary wave solution

$$
u(x, t)=\frac{-\alpha}{2 \beta}\left(1 \pm \operatorname{coth}\left[\frac{\sqrt{\rho}}{2 \sqrt{\alpha}}(x-\rho t)\right]\right) .
$$

Figures 6-8 show the behavior of the solutions (3.23), (3.24) for some arbitrary constants. 

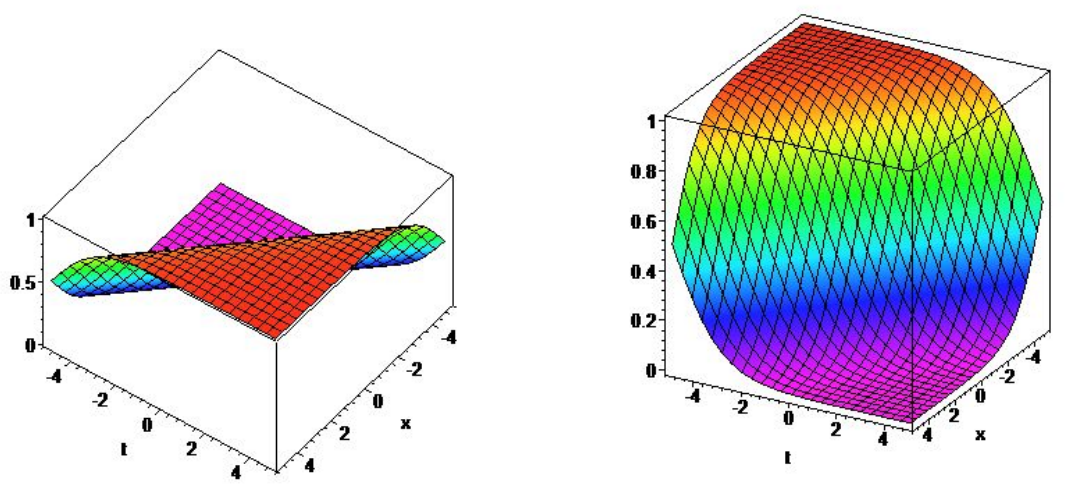

Figure 7. Solution (3.23) for $\alpha=\rho=-1, \beta=1$, respectively.
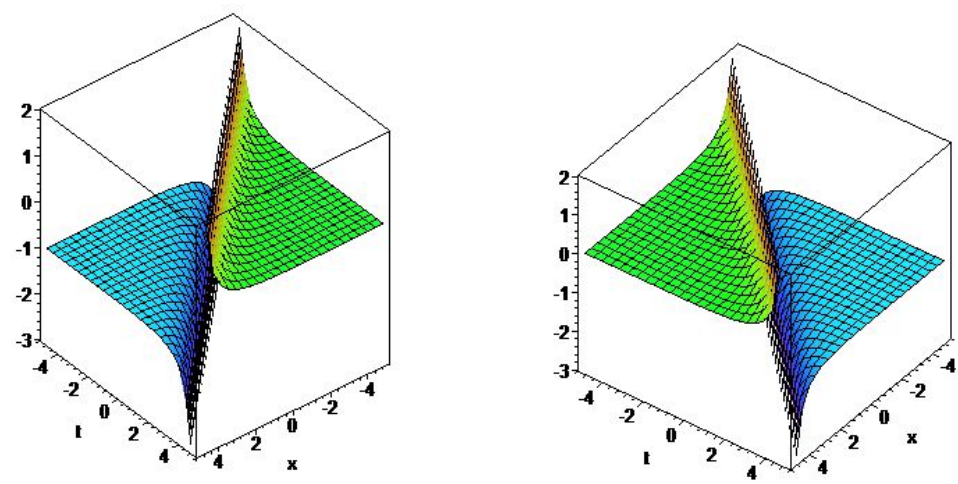

Figure 8. Solution (3.24) for $\alpha=\beta=\rho=1$, respectively.

\section{Conclusion}

In this study, the trial solution method is studied for the nonlinear partial differential equations. To this reason, the KS equation, the ZK-BBM equation and the Gardner equation have been considered. With the implementation of the trial solution algorithm to these equations for the first time, solitary wave, bright, dark and periodic exact traveling wave solutions of the considered equations have been attained.

The solutions have been checked and graphs have been given via package programs to see the behavior of the waves. Figures 1-8 show the bright and dark soliton, periodic and single kink structure of obtained solutions for some arbitrary constants. Finding hyperbolic function solutions with important physical meanings as indicated will help interpreting phenomena considered. Considering all the results and graphs demonstrates that the method is an efficient tool for NLEs. The method is a problem dependent method and according to the structure of the reduced ODE it provides several solutions such as single kink, soliton, and periodic solutions.

\section{References}

[1] Ali, A., Iqbal, M.A., Mohyud-Din, S.T.: Solitary wave solutions Zakharov-Kuznetsov-Benjamin-Bona-Mahony (ZK-BBM) equation. J. Egypt. Math. Soc. 24, 44-48 (2016).

[2] Baskonus, H.M., Altan Koc, D., Bulut, H.: New travelling wave prototypes to the nonlinear Zakharov-Kuznetsov equation with power law nonlinearity. Nonlinear Sci. Lett. A. 7 (2), 67-76 (2016).

[3] Benjamin, T.B., Bona, J.L., Mahony, J.J.: Model equations for long waves in nonlinear dispersive system. Philos. Trans. R. Soc. Lond. Ser. A. 272, 47-48 (1972). 
[4] Daoui, A.K., Triki, H.: Solitary waves, shock waves and singular solitons of Gardner's equation for shallow water dynamics. Acta Phys. Pol. B. 45 (6), 1135-1145 (2014).

[5] Demiray, S.T.: New exact solutions for generalized Gardner equation. Kuwait J. Sci. 44 (1), 1-8 (2017).

[6] Du, X.H.: An irrational trial equation method and its applications. Pramana-J. Phys. 75 (3), 415-422 (2010).

[7] Gepreel, K.A.: Explicit Jacobi elliptic exact solutions for nonlinear partial fractional differential equations. Adv. Differ. Equ-Ny. 2014:286 (2014).

[8] Guner O., Bekir, A., Cevikel, A.C.: Dark soliton and periodic wave solutions of nonlinear evolution equations. Adv. Differ. Equ-Ny. 2013:68 (2013).

[9] Hamdi, S., Morse, B., Halphen, B., Schiesser, W.: Analytical solutions of long nonlinear internal waves: Part I. Nat. Hazards. 57, 597-607 (2011).

[10] Jawad, A.J.M.: New exact solutions of nonlinear partial differential equations using Tan-Cot function method. Studies in Mathematical Sciences. 5 (2), 13-25 (2012).

[11] Kudryashov, N.A., Sinelshchikov, D.I.: Nonlinear wave in bubbly liquids with consideration for viscosity and heat transfer. Phys. Lett. A. 374, 2011-2016 (2010).

[12] Kudryashov, N.A., Sinelshchikov, D.I: Nonlinear evolution equation for describing waves in bubbly liquids with viscosity and heat transfer consideration. Appl. Math. Comput. 217, 414-421 (2010).

[13] Liu, C.S.: Using trial equation method to solve the exact solutions for two kinds of KdV equations with variable coefficients. Acta Phys. Sin. 54 (10), 4506-4510 (2005).

[14] Liu, C.S.: Trial equation method to nonlinear evolution equations with rank inhomogeneous: Mathematical discussions and its applications. Commun. Theor. Phys. 45 (2), 219-223 (2006).

[15] Liu, C.S.: A new trial equation method and its applications. Commun. Theor. Phys. 45 (3), 395-397 (2006).

[16] Lu, J.: New exact solutions for Kudryashov-Sinelshchikov equation. Adv. Differ. Equ-Ny. 2018:374 (2018).

[17] Ma, W.X., Wu, H.Y., He, J.S.: Partial differential equations possessing Frobenius integrable decompositions. Phys. Lett. A. $364,29-32(2007)$.

[18] Miura, R.M.: Korteweg-de vries equation and generalizations I. A remarkable explicit nonlinear transformation. J. Math. Phys. 9, 1202-1204 (1968).

[19] Odabasi, M., Misirli, E.: Application of the extended trial equation method to the nonlinear evolution equations. Math.Sci. Appl. E-Notes. 2 (1), 28-33 (2014).

[20] Odabasi, M., Misirli, E.: A note on the traveling wave solutions of some nonlinear evolution equations. Optik. 142, 394-400 (2017).

[21] Odabasi, M., Misirli, E.: On the solutions of the nonlinear fractional differential equations via the modified trial equation method. Math. Method Appl. Sci. 41, 904-911 (2018).

[22] Odabasi, M.: Traveling wave solutions of conformable time-fractional Zakharov-Kuznetsov and Zoomeron equations. Chin. J. Phys. 64, 194-202 (2020).

[23] Odabasi, M., Pinar, Z., Kocak, H.: Analytical solutions of some nonlinear fractional-order differential equations by different methods. Math Meth Appl Sci. 1-12 (2020). https:/ /doi.org/10.1002/mma.6313

[24] Wazwaz, A.M.: A study on KdV and Gardner equations with time-dependent coefficients and forcing terms. Appl. Math. Comput. 217, 2277-2281 (2010).

[25] Yel, G., Sulaiman, T.A., Baskonus, H.M.: On the complex solutions to the (3+1)-dimensional conformable fractional modified KdV-Zakharov-Kuznetsov equation. Mod. Phys. Lett. B. 34 (5), 2050069 (2020). 


\section{Affiliations}

MERYEM OdABAŞI KÖPRÜLÜ

AdDRESS: Ege University, Tire Kutsan Vocational School, 35900, Izmir-Turkey.

E-MAIL: meryemodabasi@gmail.com, meryem.odabasi@ege.edu.tr

ORCID ID: 0000-0002-3025-3063 